\title{
La escala de autoestima de Rosenberg: Validación para Chile en una muestra de jóvenes adultos, adultos y adultos mayores
}

\author{
Cristian A Rojas-Barahonala, Beatriz Zegers Pla, \\ Carla E Förster $\mathbf{M}^{2 \mathbf{b}}$. \\ Rosenberg self-esteem scale: \\ Validation in a representative sample \\ of Chilean adults
}

Background: Self-esteem is positively associated to the well being of people and could be a good mental health indicator. Aim: To determine the realiability and validity of the Rosenberg Self-esteem Scale in a Chilean adult sample. Material and methods: The instrument was applied to 473 subjects living in the Metropolitan Region of Santiago, evenly distributed according to gender, age, educational level and income. The Neugarten Life Satisfaction Index (LSI-A) was also applied to the sample. Results: Cronbach's alpha for reliability of the scale was 0.754 . There was no gender bias and factor analysis grouped items into two factors ( 5 positive and 5 negative). The instrument had a correlation of 0.455 with the LSI-A. Conclusions: The Rosenberg Self-esteem Scale meets the criteria for validity and reliability of a quality instrument to measure self-esteem in Chile (Rev Méd Chile 2009; 137: 791-800).

(Key w ords: Mental health; Self assessment (Psychology); Self concept)

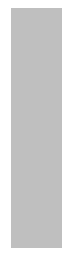

Recibido el 26 de noviembre, 2008. Aprobado el 28 de abril, 2009.

La presente investigación fue financiada por el Fondo de Ayuda a la Investigación (FAI), proyecto № PSI-001-07, de la Universidad de los Andes, Santiago, Chile.

${ }^{1}$ Escuela de Psicología, Universidad de los Andes. Santiago, Chile. ${ }^{2}$ Facultad de Educación, Pontificia Universidad Católica de Chile. Santiago, Chile.

apsicólogo

${ }^{\mathrm{b}}$ Biólogo

$\mathrm{L}$

a autoestima es un concepto ampliamente estudiado y discutido ${ }^{1-7}$, observándose su di-

Correspondencia a: Dr. Cristian A Rojas-Barahona. Escuela de Psicología, Universidad de los Andes. Av. San Carlos de Apoquindo 2200, Las Condes, Santiago, Chile. Fax: (56 2) 4129480. Teléfono: (56 2) 4129478. E mail: crojas@uandes.cl recta relación con el bienestar general de la persona $^{8}$, lo que sugiere que podría ser un buen indicador de la salud mental?. Diversas investigaciones han confirmado la relación inversa entre autoestima y síntomas depresivos ${ }^{9-11}$, obsesivos compulsivos $^{12-13}$, ansiosos ${ }^{13-15}$, entre otros. Por tanto, su conocimiento es una valiosa información que posibilitaría la predicción de posibles trastornos psicopatológicos y su prevención ${ }^{10}$. 
Existen diversos instrumentos que miden la autoestima, pero uno de los más utilizados ${ }^{16}$ es la Escala de Autoestima de Rosenberg (EAR) ${ }^{5}$. La EAR ha sido traducida y validada en distintos países e idiomas: francés ${ }^{17}$, alemán ${ }^{18}$, japonés ${ }^{19}$, portugués $^{20}$, español ${ }^{21-22}$, etc. Recientemente, Schmitt y Allik $^{23}$ realizaron un estudio multicultural, administrando la EAR en 53 naciones, en 48 de las cuales se presenta una confiabilidad sobre 0,75 .

Rosenberg 5 entiende la autoestima como un sentimiento hacia uno mismo, que puede ser positivo o negativo, el cual se construye por medio de una evaluación de las propias características. La escala fue dirigida en un principio a adolescentes, hoy se usa con otros grupos etarios. Su aplicación es simple y rápida. Cuenta con 10 ítems, divididos equitativamente en positivos y negativos (ejemplos, sentimiento positivo: "creo que tengo un buen número de cualidades", sentimiento negativo: "siento que no tengo muchos motivos para sentirme orgulloso de mi"). Es un instrumento unidimensional que se contesta en una escala de 4 alternativas, que va desde "muy de acuerdo" a "muy en desacuerdo".

La EAR es ampliamente utilizada en Chile, pero no existe evidencia de un proceso de validación publicado, por tanto el propósito de este estudio fue determinar su confiabilidad y validez en una muestra chilena.

\section{Material y MÉTODO}

Se seleccionaron 473 sujetos de la Región Metropolitana (RM) de Santiago de Chile, a partir de un muestreo intencionado por cuotas distribuidos según: escolaridad (básica, media y superior); sexo (hombre y mujer); edad (30-45, $45-59$ y 6075) e ingreso autónomo per cápita del hogar (bajo, medio, alto). La estratificación de edad consideró los estudios de Levinson ${ }^{24-25}$, que sitúan el comienzo de la Adultez Media a los 45 años, y la definición de Naciones Unidas para la Adultez Mayor, que comenzaría a los 60 años; si bien, la Adultez Joven se iniciaría en promedio a los 28 años $^{25}$, se mantuvo el rango de 15 años utilizado para delimitar los otros dos grupos etarios. La clasificación de ingreso fue acorde a la encuesta CASEN ${ }^{26}$ para la RM urbana (Bajo considera los quintiles I y II: 0-84 mil pesos; Medio los quintiles III y IV: 85-264 mil pesos, y Alto el quintil V: 265 mil pesos o más).

Se excluyó a todos los sujetos que presentaron alguna dificultad para comprender las instrucciones o que estuvieran físicamente discapacitados. La distribución de la muestra se presenta en la Tabla 1.

Se utilizó la $\mathrm{EAR}^{5}$, la cual consta de 10 afirmaciones de los sentimientos que tiene la persona sobre ella, 5 direccionadas positivamente (items 1 ,

\section{Tabla 1. Características sociodemográficas de la muestra y distribución de frecuencias de los participantes del estudio}

\begin{tabular}{|c|c|c|c|c|c|c|c|c|c|c|c|}
\hline Edad & $\begin{array}{l}\text { Ingresos } \\
\text { N. Educacional } \\
\text { Sexo }\end{array}$ & $\begin{array}{c}\text { Bajo } \\
\text { Básica }\end{array}$ & Media & Superior & $\begin{array}{l}\text { Medio } \\
\text { Básica }\end{array}$ & Media & Superior & $\begin{array}{c}\text { Alto } \\
\text { Básica }\end{array}$ & Media & Superior & $\begin{array}{l}\text { Total } \\
\text { sexo }\end{array}$ \\
\hline \multirow[t]{3}{*}{ 30-44 años } & Masculino & 10 & 10 & 6 & 11 & 10 & 10 & 7 & 11 & 10 & 85 \\
\hline & Femenino & 9 & 10 & 11 & 11 & 9 & 10 & 5 & 9 & 10 & 84 \\
\hline & Total Edad & 19 & 20 & 17 & 22 & 19 & 20 & 12 & 20 & 20 & 169 \\
\hline \multirow[t]{3}{*}{ 45-59 años } & Masculino & 10 & 10 & 5 & 8 & 10 & 9 & 4 & 10 & 10 & 76 \\
\hline & Femenino & 9 & 10 & 7 & 9 & 10 & 10 & 3 & 10 & 10 & 78 \\
\hline & Total Edad & 19 & 20 & 12 & 17 & 20 & 19 & 7 & 20 & 20 & 154 \\
\hline \multirow[t]{3}{*}{ 60-75 años } & Masculino & 10 & 7 & 4 & 9 & 11 & 9 & 6 & 9 & 10 & 75 \\
\hline & Femenino & 9 & 8 & 6 & 9 & 7 & 10 & 9 & 8 & 9 & 75 \\
\hline & Total Edad & 19 & 15 & 10 & 18 & 18 & 19 & 15 & 17 & 19 & 150 \\
\hline Total N. educacional & 57 & 55 & 39 & 57 & 57 & 58 & 34 & 57 & 59 & 473 & \\
\hline Total ingreso & & & 151 & & & 172 & & & 150 & & 473 \\
\hline
\end{tabular}


2, 4, 6 y 7) y 5 negativamente (items $3,5,8,9$ y 10). La graduación de respuestas tiene 4 puntos (1 =muy en desacuerdo, $2=$ en desacuerdo, $3=$ de acuerdo y 4 =muy de acuerdo) y se asigna el puntaje inverso a las afirmaciones direccionadas negativamente; los valores teóricos fluctúan entre 10 (baja autoestima) y 40 (alta autoestima). Es una escala autoaplicada donde los participantes marcan con una " $\mathrm{X}$ " las alternativas que más lo identifican. La EAR fue traducida desde el inglés al español y evaluada por 7 jueces expertos, tanto en idioma como en contenido, considerando una concordancia mínima de 5 de ellos (71\%) de acuerdo a los siguientes criterios: a) mantención del sentido e intencionalidad de las afirmaciones, b) lenguaje adecuado al contexto chileno, y c) revisión de aspectos formales del instrumento. Previo a la aplicación definitiva se realizó un estudio piloto con 45 sujetos para revisar la comprensión del instrumento. Se comparó la traducción con la versión original y la de Vázquez y cols ${ }^{21}$, manteniendo el sentido y orden de las afirmaciones propuesto por Rosenberg 5 . Se aplicó el Índice de Satisfacción Vital (LSI-A) de Neugarten y $\operatorname{cols}^{27-28}$ validado para Chile por Zegers, Rojas-Barahona y Förster $^{29}$, el cual consta de 20 afirmaciones. Los valores teóricos van de 0 a 20 puntos (a mayor puntaje mayor satisfacción vital). La EAR fue respondida individualmente, en un lugar silencioso, previa aceptación verbal del conocimiento informado (ver Anexo).

Estadística. Se calculó los estadísticos descriptivos del total de la EAR, para cada grupo (edad, sexo, nivel educacional y nivel de ingreso) y se analizó cada afirmación considerando: media, desviación estándar, asimetría y curtosis, correlación ítemtest, confiabilidad si el ítem era eliminado y correlación con el puntaje total. La confiabilidad se estimó con Alfa de Cronbach. Para estimar posibles diferencias por edad, nivel educacional e ingresos, se utilizó ANOVA y como test a posteriori Tukey b o Tamhane, según el resultado del test de Levene. Las diferencias y sesgo de sexo se estimaron con Prueba t y con tabla de contingencia (Puntaje total EAR * Frecuencia hombres y mujeres en dichos puntajes). Se aplicó $\chi^{2}$ para establecer la independencia entre el sexo y el puntaje total obtenido. Se calculó los percentiles para el grupo total, los grupos de edades y sexo.
Con Análisis Factorial de Componentes Principales (AFCP) se determinó la validez de constructo del instrumento, considerando los requisitos del test de Kaiser-Meyer-Olkin (KMO) y el test de Bartlett. El número de factores se estableció incorporando los autovalores superiores a 1 . Se utilizó el método de normalización Varimax y se analizaron los valores de los pesos factoriales rotados, confirmando que su agrupación correspondiera a los factores teóricos del instrumento. La validez concurrente se estimó con un análisis de correlación de Pearson entre los puntajes de la EAR y el LSI-A.

\section{Resultados}

Los puntajes obtenidos por los sujetos fluctuaron entre 18 y 40 puntos. La media general fue de 32,47 puntos (ds $=4,14)$, similar a la observada en cada grupo de edad, donde no hubo diferencias significativas $\left(\mathrm{F}_{(2,470)}=0,431 ; \mathrm{p}=0,650\right)$. Las varianzas de los grupos fueron heterogéneas $\left(\mathrm{F}_{(2,470)}=3.641\right.$; $\mathrm{p}=0,027)$. El grupo de edad de $60-75$ años presentó el mínimo más bajo (18 puntos, Tabla 2).

El análisis por sexo mostró un rango de respuesta menor en hombres (23-40 puntos), y una desviación estándar significativamente mayor en mujeres $\left(\mathrm{ds}=4.395 ; \mathrm{F}_{(1,471)}=3.968 ; \mathrm{p}=0,047\right)$, no obstante, no hubo diferencias en las medias del puntaje total de autoestima de hombres y mujeres $\left(\mathrm{t}_{(471)}=-1.890 ; \mathrm{p}=0,059\right.$, Tabla 2).

El análisis por nivel educacional mostró un ascenso en las medias a mayor escolaridad, y la diferencia se encontró entre quienes cursaron enseñanza básica y los otros dos grupos $\left(\mathrm{F}_{(2,470)}\right.$ $=14.373 ; \mathrm{p}=0,000)$. Las varianzas de los grupos fueron homogéneas $\left(F_{(2.470)}=0,260 ; p=0,771\right) y$ el rango más amplio de puntajes se encontró en el nivel superior (18-40, Tabla 2).

El resultado según el nivel de ingresos indicó que hubo un aumento en la media a medida que el ingreso fue mayor $\left(\mathrm{F}_{(2,470)}=12.634 ; \mathrm{p}=0,000\right)$, donde la diferencia radica entre el nivel alto con los otros dos $(\mathrm{p}<0,05)$. El rango más amplio de puntajes se encontró en el nivel bajo (18-40), pero en todos los grupos se alcanzó el máximo (Tabla 2).

En la Tabla 3 se presentan las medias y desviaciones estándar de cada afirmación según sexo, observándose similitud en ambos grupos, 
Tabla 2. Estadísticos descriptivos y confiabilidad del puntaje total por cada grupo de edad, sexo, nivel educacional e ingresos

\begin{tabular}{|llllllll|}
\hline Variable & Categoría & N & Media & d.s. & M ínimo & M áximo & Confiabilidad \\
\hline \multirow{2}{*}{ Edad } & $30-44$ & 32 & 32,37 & 4.399 & 22 & 40 &, 789 \\
& $45-59$ & 33 & 32,73 & 3.751 & 22 & 40 &, 708 \\
\multirow{2}{*}{ Sexo } & $60-75$ & 32 & 32,33 & 4.221 & 18 & 40 &, 751 \\
& Mujer & 237 & 32,83 & 4.395 & 18 & 40 &, 736 \\
\multirow{5}{*}{ Nivel educacional } & Hombre & 236 & 32,11 & 3.834 & 23 & 40 &, 764 \\
& Básica & 148 & 31,08 & 3.932 & 22 & 40 &, 708 \\
\multirow{4}{*}{ Ingreso } & Media & 169 & 32,74 & 4.096 & 22 & 40 &, 729 \\
& Superior & 156 & 33,51 & 4.030 & 18 & 40 &, 781 \\
\multirow{2}{*}{ Total } & Bajo & 151 & 31,45 & 4.386 & 18 & 40 &, 746 \\
& Medio & 172 & 32,26 & 3.706 & 22 & 40 &, 691 \\
& Alto & 150 & 33,75 & 4.037 & 22 & 40 &, 785 \\
& & 473 & 32,47 & 4.136 & 18 & 40 &, 754 \\
\hline
\end{tabular}

Tabla 3. Estadísticos descriptivos y comparación de medias entre hombres y mujeres en cada ítem

\begin{tabular}{|c|c|c|c|c|c|c|c|c|c|c|}
\hline \multicolumn{2}{|c|}{ Ítems } & \multirow{2}{*}{$\begin{array}{l}\text { Media } \\
3,52\end{array}$} & \multirow{2}{*}{$\begin{array}{l}\text { d.s. } \\
0,51\end{array}$} & \multirow{2}{*}{$\begin{array}{c}\text { Hombres } \\
\text { Asimetría } \\
-0,17\end{array}$} & \multirow{2}{*}{$\begin{array}{l}\text { Curtosis } \\
-1,74\end{array}$} & \multirow{2}{*}{$\begin{array}{l}\text { Media } \\
3,61\end{array}$} & \multirow{2}{*}{$\begin{array}{l}\text { d.s. } \\
0,55\end{array}$} & \multirow{2}{*}{$\begin{array}{c}\text { M ujeres } \\
\text { Asimetría } \\
-1,36\end{array}$} & \multirow{2}{*}{$\begin{array}{c}\text { Curtosis } \\
2,59\end{array}$} & \multirow{2}{*}{$\frac{\mathbf{t}}{-1,94}$} \\
\hline 1. & $\begin{array}{l}\text { Siento que soy una persona digna de } \\
\text { aprecio, al menos en igual medida que } \\
\text { los demás. }\end{array}$ & & & & & & & & & \\
\hline 2. & $\begin{array}{l}\text { Creo que tengo un buen número de } \\
\text { cualidades. }\end{array}$ & 3,33 & 0,53 & 0,11 & $-0,80$ & 3,48 & 0,58 & $-0,70$ & 0,27 & $-2,86^{*}$ \\
\hline 3. & $\begin{array}{l}\text { En general, me inclino a pensar que soy } \\
\text { un/a fracasado/a. }\end{array}$ & 3,42 & 0,68 & $-1,17$ & 1,75 & 3,49 & 0,70 & $-1,54$ & 2,64 & $-1,03$ \\
\hline 4. & $\begin{array}{l}\text { Soy capaz de hacer las cosas tan bien } \\
\text { como la mayoría de la gente. }\end{array}$ & 3,42 & 0,55 & $-0,21$ & $-0,95$ & 3,48 & 0,62 & $-0,98$ & 0,97 & $-1,06$ \\
\hline 5. & $\begin{array}{l}\text { Siento que no tengo muchos motivos } \\
\text { para sentirme orgulloso/a de mí. }\end{array}$ & 2,97 & 0,91 & $-0,59$ & $-0,45$ & 3,01 & 1,06 & $-0,78$ & $-0,64$ & $-0,42$ \\
\hline 6. & Tengo una actitud positiva hacia mí mismo/a. & 3,36 & 0,61 & $-0,64$ & 0,62 & 3,48 & 0,69 & $-1,28$ & 1,46 & $-1,94$ \\
\hline & $\begin{array}{l}\text { En general, estoy satisfecho/a conmigo } \\
\text { mismo/a. }\end{array}$ & 3,27 & 0,63 & $-0,38$ & $-0,12$ & 3,42 & 0,72 & $-1,23$ & 1,52 & $-2,36^{*}$ \\
\hline 8. & Desearía valorarme más a mí mismo/a. & 2,14 & 0,92 & 0,54 & $-0,48$ & 2,19 & 1,03 & 0,46 & $-0,92$ & $-0,56$ \\
\hline & $\begin{array}{l}\text { A veces me siento verdaderamente inútil. } \\
\text { A veces pienso que no soy }\end{array}$ & 3,28 & 0,80 & $-0,86$ & $-0,01$ & 3,28 & 0,85 & $-0,95$ & 0,04 & 0,07 \\
\hline & bueno/a para nada & 3,39 & 0,75 & $-1,08$ & 0,66 & 3,40 & 0,78 & $-1,15$ & 0,67 & $-0,10$ \\
\hline Tota & l escala & 32,11 & 3,83 & 0,02 & $-0,63$ & 32,83 & 4,40 & $-0,31$ & $-0,30$ & $-1,89$ \\
\hline
\end{tabular}

${ }^{*} \mathrm{p}<0,05$. 
con excepción de los ítems 2 y 7 , donde las diferencias son estadísticamente significativas. La asimetría de cada afirmación fue similar en ambos sexos, con una tendencia leve hacia el lado izquierdo tanto en hombres $(0,54$ y $-1,17)$ como en mujeres $(0,46$ y $-1,54)$ (afirmaciones 8 y 3 , respectivamente). Los valores de curtosis estuvieron en rangos similares de normalidad con excepción de los ítems 1, 4 y 7, que tuvieron valores de tendencia inversa en hombres y mujeres.

El análisis de $\chi^{2}$ que compara las frecuencias de ocurrencia de hombres y mujeres en cada decil de la distribución de puntajes totales de autoestima, indica que no hubo diferencias estadísticamente significativas $\left(\chi_{(9)}^{2}=10.901 ; \mathrm{p}\right.$ $=0,283$ ).

En los datos normativos para el grupo total, por edades y por sexo, se observó que los valores fueron similares para todos los percentiles, con excepción del P1, donde el grupo 60-75 años presentó un puntaje total de 3 puntos menos que los otros grupos. En el P99 todos los grupos alcanzaron el puntaje máximo (Tabla 4).

En la Tabla 5 se presentó el análisis general de la EAR. Destacaron los ítems 5 y 8 con las medias más bajas (2,99 y 2,16, respectivamente) y el ítem 1 con la media más alta y la menor desviación estándar $(\overline{\mathrm{x}}=3,56$; ds $=0,533)$. La asimetría y la curtosis de los ítems, si bien presentaron desviaciones respecto de una distribución normal, de acuerdo con los valores de los errores típicos de ambas medidas, se consideraron en un rango de normalidad ( $\mathrm{a}=-0,138$, error $=0,112 ; \mathrm{k}=-0,439$, error $=0,224)$. La correlación ítem-test fue significativa $(\mathrm{p}<0,001)$ y superior a 0,5 en todos los ítems, con excepción del ítem $1(0,458)$ y la correlación con el LSI-A fue positiva y significativa ( $p<0,001$ ) en todas las otras afirmaciones, siendo la 1 y la 5 las que presentaron los valores más bajos $(0,16$ y 0,18 , respectivamente). La correlación entre autoestima y satisfacción vital indica que hubo una relación lineal y directa entre ambos constructos ( $r=0,455$; $p<0,001$, Tabla 4).

La correlación entre la EAR y LSI-A fue similar entre hombres y mujeres $(0,465$ y 0,453 , respectivamente). Al analizar la relación por grupos de edad, se observaron valores equivalentes entre ellos (3044 años, $r=0,468$; $45-59$ años, $r=0,455$ y 60-75 años, $r=0,449$ ). Hubo diferencias en el nivel educacional, donde la mayor correlación se alcanzó en el nivel Medio $(0,491)$ y el valor más bajo en el Superior $(0,398)$. El nivel Básico presentó un valor de 0,415. Para los grupos de ingreso per cápita Bajo y Medio, los coeficientes fueron similares $(0,473$ y 0,483 , respectivamente), mientras que para el ingreso Alto este coeficiente cayó a 0,288.

Se estimó una confiabilidad de $\alpha=0,754$ para el total de la muestra. En la Tabla 4 se presentó la correlación ítem-test, que fluctuó entre 0,46 y 0,67 ( $p<0,001)$. Al realizar el análisis por edad, en las

Tabla 4. D atos normativos del puntaje total, por edad y por sexo

\begin{tabular}{|ccccccc|}
\hline Percentiles & Total & $\mathbf{3 0 - 4 4}$ & $\mathbf{4 5 - 5 9}$ & $\mathbf{6 0 - 7 5}$ & H ombres & M ujeres \\
\hline 1 & 23 & 23 & 23 & 20 & 23 & 22 \\
10 & 27 & 26 & 28 & 28 & 27 & 27 \\
20 & 29 & 28 & 29 & 29 & 29 & 29 \\
30 & 30 & 30 & 31 & 29 & 30 & 30 \\
40 & 31 & 31 & 32 & 31 & 31 & 32 \\
50 & 32 & 32 & 33 & 32 & 32 & 33 \\
60 & 34 & 33 & 34 & 34 & 33 & 34 \\
70 & 35 & 35 & 35 & 35 & 34 & 35 \\
80 & 37 & 37 & 36 & 36 & 36 & 37 \\
90 & 38 & 39 & 37 & 38 & 37 & 39 \\
99 & 40 & 40 & 40 & 40 & 40 & 40 \\
N total & 473 & 169 & 154 & 150 & 236 & 237 \\
\hline
\end{tabular}


Tabla 5. Estadísticos descriptivos de los ítems de la Escala de Autoestima de Rosenberg

\begin{tabular}{|c|c|c|c|c|c|c|c|}
\hline & Media & d.s. & Asimetría & Curtosis & $\begin{array}{l}\text { Correlación } \\
\text { ítem-test (1) }\end{array}$ & $\begin{array}{l}\text { Alfa si se } \\
\text { elimina } \\
\text { el ítem }\end{array}$ & $\begin{array}{l}\text { Correlación } \\
\text { con LSI-A }\end{array}$ \\
\hline $\begin{array}{l}\text { 1. Siento que soy una persona digna de } \\
\text { aprecio, al menos en igual medida que } \\
\text { los demás. }\end{array}$ & 3,56 &, 533 &,- 806 &, 534 &, $458^{* *}$ &, 743 &, $160^{* * *}$ \\
\hline $\begin{array}{l}\text { 2. Creo que tengo un buen número de } \\
\text { cualidades. }\end{array}$ & 3,40 &, 560 &,- 307 &,- 383 &, $535^{* *}$ &, 734 &, $204^{* *}$ \\
\hline $\begin{array}{l}\text { 3. En general, me inclino a pensar que soy } \\
\text { un/a fracasado/a. }\end{array}$ & 3,46 & ,691 & -1.353 & 2.145 &, $644^{* *}$ & ,718 &, $292 *$ \\
\hline $\begin{array}{l}\text { 4. Soy capaz de hacer las cosas tan bien } \\
\text { como la mayoría de la gente. }\end{array}$ & 3,45 & ,588 &,- 647 & 169 &, $505^{* *}$ &, 738 &, $248^{* * *}$ \\
\hline $\begin{array}{l}\text { 5. Siento que no tengo muchos motivos para } \\
\text { sentirme orgulloso/a de mi. }\end{array}$ & 2,99 & ,988 &,- 701 &,- 543 &, $508^{* *}$ & ,759 &, $180^{* *}$ \\
\hline $\begin{array}{l}\text { 6. Tengo una actitud positiva hacia } \\
\text { mí mismo/a. }\end{array}$ & 3,42 & ,657 &,- 976 & ,980 &, $619^{* *}$ &, 722 &, $323^{* *}$ \\
\hline $\begin{array}{l}\text { 7. En general, estoy satisfecho conmigo } \\
\text { mismo/a. }\end{array}$ & 3,34 & ,678 &,- 837 & ,689 &, $592^{* *}$ &, 727 &, $404^{* *}$ \\
\hline 8. Desearía valorarme más a mí mismo/a. & 2,16 & 978 & ,498 &,- 723 &, $500^{* *}$ &, 760 &, $225^{* *}$ \\
\hline 9. A veces me siento verdaderamente inútil. & 3,28 & ,823 &,- 905 &, 019 &, $656^{* *}$ &, 717 &, $313^{* *}$ \\
\hline $\begin{array}{l}\text { 10. A veces pienso que no soy bueno/a } \\
\text { para nada. }\end{array}$ & 3,39 &, 763 & -1.116 &, 652 &, $671^{* *}$ &, 714 &, $254^{* *}$ \\
\hline Total escala & 32,47 & 4,136 &,- 138 &,- 439 & 1 & &, $455^{* *}$ \\
\hline
\end{tabular}

(1) La correlación ítem-test fue calculada considerando la escala completa

**:La correlación es significativa al nivel 0,01 (bilateral).

categorías 30-44 años y 60-75 años se mantuvo la confiabilidad dentro del rango esperado $(0,789 \mathrm{y}$ 0,751, respectivamente); para el grupo 45-59 años la confiabilidad disminuyó a 0,708 . Esta situación se puede explicar por la menor dispersión de los puntajes totales obtenidos por los sujetos, ya que al disminuir la variabilidad, la confiabilidad del instrumento también disminuye; no obstante, el valor de la confiabilidad se consideró aceptable para este tipo de escalas.

La confiabilidad fue similar en hombres y mujeres $(0,736$ y 0,764 , respectivamente). En el nivel educacional se observó un aumento creciente de alfa (Básico =0,708; Medio =0,729 y Superior $=0,781$ ), mientras que en el nivel de ingresos el valor más bajo se dio en el nivel Medio $(0,691)$, los otros niveles fueron similares entre sí, igual que en los grupos de edad (Tabla 2). La baja confiabilidad del nivel Medio puede atribuirse a la menor dispersión de puntajes de esos segmentos.

En el AFCP se observó que los ítems de la escala se agrupan en dos factores, explicando el $51,718 \%$ de la varianza total de los puntajes obtenidos por los sujetos (Tabla 6). La varianza explicada por cada factor luego de la rotación fue de $27,476 \%$ en el primer componente y de $24,242 \%$ en el segundo.

La matriz de componentes rotados (Tabla 7) muestra los pesos que tiene cada afirmación en el componente al que se asocia. Las afirmaciones $1,2,4,6,7$ se agrupan en el primer componente, mientras que las afirmaciones 3,5 , 
Tabla 6. Varianza total explicada

\begin{tabular}{|c|c|c|c|c|c|c|c|c|c|}
\hline \multirow[t]{2}{*}{ Componente } & \multicolumn{3}{|c|}{ Autovalores iniciales } & \multicolumn{3}{|c|}{$\begin{array}{l}\text { Sumas de las saturaciones al } \\
\text { cuadrado de la extracción }\end{array}$} & \multicolumn{3}{|c|}{$\begin{array}{l}\text { Suma de las saturaciones al } \\
\text { cuadrado de la rotación }\end{array}$} \\
\hline & Total & $\begin{array}{l}\% \text { de la } \\
\text { varianza }\end{array}$ & $\begin{array}{c}\% \\
\text { acumulado }\end{array}$ & Total & $\begin{array}{l}\% \text { de la } \\
\text { varianza }\end{array}$ & $\begin{array}{c}\% \\
\text { acumulado }\end{array}$ & Total & $\begin{array}{l}\% \text { de la } \\
\text { varianza }\end{array}$ & $\begin{array}{c}\% \\
\text { acumulado }\end{array}$ \\
\hline 1 & 3.392 & 33.924 & 33.924 & 3.392 & 33.924 & 33.924 & 2.748 & 27.476 & 27.476 \\
\hline 2 & 1.779 & 17.794 & 51.718 & 1.779 & 17.794 & 51.718 & 2.424 & 24.242 & 51.718 \\
\hline 3 & ,933 & 9.329 & 61.047 & & & & & & \\
\hline 4 & 865 & 8.649 & 69.696 & & & & & & \\
\hline 5 &, 761 & 7.613 & 77.309 & & & & & & \\
\hline 6 & ,588 & 5.882 & 83.190 & & & & & & \\
\hline 7 &, 581 & 5.815 & 89.005 & & & & & & \\
\hline 8 &, 502 & 5.024 & 94.030 & & & & & & \\
\hline 9 & ,333 & 3.332 & 97.362 & & & & & & \\
\hline 10 & ,264 & 2.638 & 100.000 & & & & & & \\
\hline
\end{tabular}

Método de extracción: Análisis de componentes principales.

Tabla 7. M atriz de componentes rotados(a)

\begin{tabular}{|c|c|c|c|}
\hline \multicolumn{2}{|c|}{ ÍTEM } & \multicolumn{2}{|c|}{ Componente } \\
\hline & & 1 & 2 \\
\hline 1. & $\begin{array}{l}\text { Siento que soy una persona digna de aprecio, al menos en igual medida } \\
\text { que los demás. }\end{array}$ & 0,647 & \\
\hline 2. & Creo que tengo un buen número de cualidades. & 0,685 & \\
\hline 3. & En general, me inclino a pensar que soy un/a fracasado/a. & & 0,666 \\
\hline 4. & Soy capaz de hacer las cosas tan bien como la mayoría de la gente. & 0,729 & \\
\hline 5. & Siento que no tengo muchos motivos para sentirme orgulloso/a de mi. & & 0,634 \\
\hline 6. & Tengo una actitud positiva hacia mí mismo/a. & 0,780 & \\
\hline 7. & En general, estoy satisfecho conmigo mismo/a. & 0,773 & \\
\hline 8. & Desearía valorarme más a mí mismo/a. & & 0,494 \\
\hline 9. & A veces me siento verdaderamente inútil. & & 0,805 \\
\hline & A veces pienso que no soy bueno/a para nada. & & 0,789 \\
\hline & Alfa de Cronbach & 0,786 & 0,705 \\
\hline \multicolumn{2}{|c|}{ Porcentaje de varianza explicado } & 27.476 & 24.242 \\
\hline
\end{tabular}

Método de extracción: Análisis de componentes principales. Método de rotación: Normalización Varimax con Kaiser. (a) La rotación ha convergido en 3 iteraciones.

8, 9 y 10 se agrupan en el segundo componente. Esta configuración concuerda con la direccionalidad de las afirmaciones, donde el componente 1 tiene un sentido positivo respec- to de la autoestima de la persona y el componente 2 corresponde a las afirmaciones que tienen un sentido negativo. La consistencia interna de cada factor expresada a través de alfa 
de Cronbach fue de 0,786 para el Componente 1 y de 0,705 para el Componente 2 .

\section{DisCUSIÓN}

La medición de la autoestima puede ser de gran utilidad para predecir o prevenir trastornos psicopatológicos ${ }^{10}$, aunque su operacionalización no es fácil de lograr, generándose cuestionamientos metodológicos a los instrumentos utilizados ${ }^{30}$. En este sentido, la EAR ha sido ampliamente estudiada en términos de constructo (por ejemplo, 17-18) y las opiniones son divergentes. A continuación se discuten los puntos críticos y el comportamiento de la EAR en la muestra chilena. En cuanto a la confiabilidad el valor fue de 0,754 para el total de la muestra, encontrándose dentro del rango esperado para este instrumento ${ }^{23}$, que fluctúa entre 0,72 y 0,89 .

Con respecto a la normalidad de las respuestas se observa una tendencia hacia una alta autoestima, evidenciándose la media más alta en los ítems positivos y más baja en los ítems negativos, situación similar a lo expresado por en la mayoría de las validaciones (por ejemplo, 17, 22, 30). No obstante, en el presente estudio la escala total se mantiene en los rangos de normalidad en asimetría y curtosis, lo que permite hacer un análisis factorial exploratorio sin necesidad de transformar los datos originales.

La media registrada en este estudio $(\overline{\mathrm{x}}=32,47)$, para la Región Metropolitana, es similar a la reportada en otro estudio chileno, también en población no clínica $(\overline{\mathrm{x}}=33,12)$ y al promedio general que se observa en 53 naciones $(\bar{x}=30,85)^{23}$. En este último estudio destacan medias bajas en algunas zonas específicas, especialmente en países asiáticos, como por ejemplo Japón $(\overline{\mathrm{x}}=25,50)$. Los datos normativos de la muestra total no varían por edad ni por sexo, sin embargo, hay un número importante de casos que obtienen los puntajes más altos ( 55 casos), lo que dificultaría la diferenciación de los sujetos en el rango superior de la escala, esto se reporta también en Alemania ${ }^{18}$.

Los resultados indican que los pesos factoriales de los ítems positivos cargan el primer factor, mientras que los ítems negativos tienen mayor peso en el segundo, coincidiendo con Rosenberg $^{5}$, Gana y cols ${ }^{17}$ y Martín-Albo y cols ${ }^{22}$. Hay que dejar claro que aun cuando el análisis muestra dos factores, la naturaleza de las respuestas hace suponer que la escala mide solo una dimensión conceptual. El ítem 5 es el único que discrepa con otros estudios 23 en cuanto a su peso factorial $(0,24)$, siendo más estable en este caso $(0,63)$.

El análisis de las variables sociodemográficas muestra similitudes con otros estudios $9,18,22$ en cuanto a la ausencia de diferencias por sexo y la existencia de diferencias asociadas a factores educacionales. No obstante, en la variable edad los resultados no concuerdan con los estudios mencionados, dada la ausencia de diferencia entre los grupos etarios. Esto puede explicarse porque las diferencias reportadas se dan entre adolescentes y adultos 9 , y en el presente estudio se trabaja sólo con adultos. Con respecto al nivel de ingreso y educacional se observa una relación directa, es decir quienes tienen salarios bajos y menores estudios presentan una menor autoestima.

En conclusión, se puede señalar que la EAR cumple con los criterios de validez y confiabilidad de un instrumento de calidad para medir la autoestima en Chile y su comportamiento es similar a lo esperado por Rosenberg ${ }^{5-6}$ para la población original. Si bien en el presente estudio este instrumento responde adecuadamente a la mayoría de los cuestionamientos metodológicos, sería recomendable profundizar su investigación realizando un análisis factorial confirmatorio y construir baremos que permitan evaluar casos clínicos únicos y su evolución en los programas de tratamiento. Del mismo modo, no existe acuerdo en la categorización de lo que se considera baja, media y alta autoestima (ver 31-32), por lo que se requieren estudios empíricos que permitan definir estas categorías.

\section{Agradecimientos}

De manera especial agradecemos los aportes de AJ Vázquez Morejón y la valiosa colaboración de Claudia Cassis, Macarena Gallegos, Francisco León, Andrea Pino y Diego Ribalta. 


\section{REFERENCIAS}

1. Chen G, Gully S M, Eden D. General self-efficacy and self-esteem: Toward theoretical and empirical distinction between correlated self-evaluations. J Org Behav 2004; 25: 375-95.

2. Coopersmith S. The antecedents of self-esteem. San Francisco: Editorial Freedman \& Company; 1967.

3. Francis LJ, Wilcox C. Self-esteem: Coopersmith and Rosenberg compared. Psychol Rep 1995; 76: 1050.

4. Lightsey OR, Burke M, Ervin A, Henderson D, Yee C. Generalized Self-Efficacy, Self-Esteem, and Negative Affect. Canad J Behav Scien 2006; 38: 72-80.

5. Rosenberg M. Society and the adolescent selfimage. Princeton: Princeton University Press; 1965.

6. Rosenberg M, Schooler C, Schoenbach C, Rosenberg F. Global self-esteem and specific self-esteem. Amer Socio Rev 1995; 60: 141-56.

7. Thompson AH, Barnsley RH, Battle J. The relative age effect and the development of self-esteem. Educ Res 2004; 46: 313-20.

8. Robis RW, Hendin HM, Trzesniewsini KH. Measuring global self-esteem: construct validation of a single item measure and the Rosenberg SelfEsteem Scale. Pers Soc Psychol Bull 2001; 27: 151-61.

9. Garaigordobil M, Pérez Ji, Mozaz M. Self-concept, self-esteem and psychopathological symptoms. Psicothema 2008; 20: 114-23.

10. FAN F, Fu J. Self-concept and mental health of college students. Chin Ment Heal J 2001; 15: 76-7.

11. Кıм YH. Correlation of mental health problems with psychological constructs in adolescence: Final results from a 2-year study. Int J Nurs Stud 2003; 40: 115-24.

12. Biby EL. The relation between body dysmorphic disorder and depression, self-esteem, somatization and obsessive-compulsive disorder. J Clin Psychol 1998; 54: 489-99.

13. Bohne A, Keuthen NJ, Wilhelm S, Deckersback T, Jenike MA. Prevalence of symptoms of body dysmorphic disorder and its correlates: A crosscultural comparison. Psychosomatics: J Consultation Liasion Psychol 2002; 43: 486-90.

14. Fickova E. Personality dimensions and self-esteem indicators relationships. Studia Psychol 1999; 41: 323-8.

15. Newbegin I, Owens A. Self-esteem and anxiety in secondary school achievement. J Soc Behav and Pers 1996; 11: 521-30.
16. Byrne BM. Measuring self-concept across the life-span: Issues and instrumentation. Washington, DC: American Psychological Association; 1996.

17. Vallieres EF, Vallerand RJ. Traduction et validation Canadienne-Francaise de l’Echelle de l'Estime de soi de Rosenberg. Intern J Psychol 1990; 25: 305-16.

18. Roth M, Decker O, Yorck Herzberg P, Brähler E. Dimensionality and Norms of the Rosenberg Self-esteem Scale in a German General Population Sample. Europ J Psychol Assess 2008; 24: 190-7.

19. Окаda T, Nagai T. Self-esteem and anthrophobictendency in adolescents. Japan J Psychol 1990; 60: 386-9.

20. Santos PJ, Maia J. Análise factorial confirmatória e validaçao preliminar de uma versão portuguesa da escala de auto-estima de Rosenberg. Psicología: Teoría, Investigaçao e Práctica 2003; 2: 25368.

21.VÁzquez AJ, Jiménez R, VÁzquez-Morejón R. Escala de autoestima de Rosenberg: fiabilidad y validez en población clínica española. Apuntes de Psicol 2004; 22: 247-55.

22. Martn-Albo J, Núñez Jl, Navarro JG, Grijalvo F. The Rosenberg Self-Esteem Scale: Translation and Validation in University Students. Span J Psychol 2007; 10: 458-67.

23. Sснмitt DP, Allik J. Simultaneous Administration of the Rosenberg Self-Esteem Scale in 53 Nations: Exploring the Universal and Culture-Specific Features of Global Self-Esteem. J Pers Soc Psychol 2005; 89: 623-42.

24. Levinson D. The seasons of man's life. New York: Alfred Knopf; 1978.

25. Levinson D, Levinson J. The seasons of a woman's life. New York: Alfred Knopf; 1996.

26. CASEN. Módulos de Ingresos: Cuadros Ingresos 2003. Disponible en: http://www.mideplan.cl/ casen/modulo_ingresos.html. [Consultado el 14 de mayo de 2007]

27. Neugarten BL, Havighurst RJ, Tobin SS. The measurement of life satisfaction. J Geront 1961; 16: $134-43$.

28. Neugarten BL, Havighurst RJ, Tobin SS. The measurement of life satisfaction. En Neugarten, D.A., editor. Chicago: The University of Chicago Press; 1996; 296-323.

29. Zegers B, Rojas-Barahona CA, Föster CE. Validez y confiabilidad del Índice de Satisfacción Vital (LSI-A) de Neugarten, Havighrust \& Tobin en una muestra de adultos y adultos mayores en Chile. Ter Psicol 2009; en prensa.

30. Tomás JM, Olíver A. Análisis psicométrico confirmatorio de una medida multidimensional del 
autoconcepto en español. Interam J Psychol 2004; 38: 285-93.

31. Burns DD. Autoestima en 10 días. México: Editorial Paidós.
32. Zavala M, Vidal D, Castro M, Quiroga P, Klassen G. Funcionamiento social del adulto mayor. Cienc Enferm 2006; XII: 53-62.

\section{Anexo \\ Escala de Autoestima de Rosenberg}

A continuación encontrará una lista de afirmaciones en torno a los sentimientos o pensamientos que tiene sobre usted. Marque con una X la respuesta que más lo identifica.

\begin{tabular}{|c|c|c|c|c|}
\hline & $\begin{array}{l}\text { Muy de } \\
\text { acuerdo }\end{array}$ & $\begin{array}{c}\text { De } \\
\text { acuerdo }\end{array}$ & $\begin{array}{c}\text { En } \\
\text { desacuerdo }\end{array}$ & $\begin{array}{c}\text { Muy en } \\
\text { desacuerdo }\end{array}$ \\
\hline $\begin{array}{l}\text { 1. Siento que soy una persona digna de aprecio, } \\
\text { 2. Creo que tengo un buen número de cualida- } \\
\text { des. } \\
\text { 3. En general, me inclino a pensar que soy un } \\
\text { fracasado/a. } \\
\text { 4. Soy capaz de hacer las cosas tan bien como la } \\
\text { mayoría de la gente. } \\
\text { 5. Siento que no tengo muchos motivos para } \\
\text { 6. Tengo una actitud positiva hacia mi mismo/a. } \\
\text { 7. En general, estoy satisfecho conmigo mismo/a. } \\
\text { 8. Desearía valorarme más a mí mismo/a. } \\
\text { 9. A veces me siento verdaderamente inútil. } \\
\text { 10. A veces pienso que no soy bueno/a para } \\
\text { nada. }\end{array}$ & & & & \\
\hline
\end{tabular}

